\title{
Endovascular Stent Grafting for Recurrent Strokes Due to Fragile Innominate Artery Plaque: A Case Report
}

\author{
Takaaki IsHIKAWA, ${ }^{1}$ Tomosato YAMAZAKI, ${ }^{1}$ Masataka SATO, ${ }^{2}$ Noriyuki KATO, ${ }^{1}$ \\ Eiichi ISHIKAWA, ${ }^{3}$ Yuji MATSUMARU, ${ }^{3}$ and Akira MATSUMURA ${ }^{3}$ \\ ${ }^{1}$ Department of Neurosurgery, National Hospital Organization, Mito Medical Center, \\ Ibaraki, Ibaraki, Japan \\ ${ }^{2}$ Department of Cardiovascular Surgery, National Hospital Organization, Mito Medical \\ Center, Ibaraki, Ibaraki, Japan \\ ${ }^{3}$ Department of Neurosurgery, Faculty of Medicine, University of Tsukuba, Tsukuba, \\ Ibaraki, Japan
}

\begin{abstract}
Here we describe a case of recurrent ischemic strokes due to fragile innominate artery plaque successfully treated using endovascular stent grafting. An 80-year-old man presented with a history of recurrent strokes that were refractory to medical treatment. Computed tomography and magnetic resonance images of the thorax revealed a gross intramural plaque in the innominate artery. He was successfully treated using endovascular stent grafting. An AFX stent graft device was used to prevent further embolic strokes. The AFX stent graft has a unique endoskeleton design with a thin-walled expanded polytetrafluoroethylene fabric-known as active sealing structure-attached to the implant. Postoperatively, the patient has experienced no recurrent strokes in over 2 years of follow-up. The stent grafting procedure could be an optimal treatment option for treating fragile innominate artery plaques.
\end{abstract}

Keywords: innominate artery, fragile plaque, recurrent stroke, endovascular stent grafting, magnetizationprepared rapid acquisition gradient echo

\section{Introduction}

Stenotic lesions of the supraaortic vessels are a major cause of ischemic stroke resulting from either hemodynamic compromise or an artery-to-artery embolism. Regarding extracranial carotid artery stenosis, there have been some data on interventions such as carotid endoarterectomy or carotid artery stenting for the prevention of further ischemic strokes. ${ }^{1)}$ However, as data of stenotic lesion in the innominate artery (IA) are scarce, the natural course is not well known and the optimal treatment indications as well as the strategy are not established. ${ }^{2)}$ Particularly, embolic strokes originating from IA plaque are very rarely seen as stroke etiology. Herein, we report a case with repeated stroke originating

Received January 15, 2020; Accepted April 13, 2020

Copyright $\subseteq 2021$ by The Japan Neurosurgical Society This work is licensed under a Creative Commons AttributionNonCommercial-NoDerivatives International License. from an intraluminal plaque in the IA, successfully treated with endovascular stent grafting. The patient's wife provided written consent for this report.

\section{Case Report}

An 80-year-old man with a long history of hypercholesterolemia and diabetes mellitus presented at our hospital with an abrupt onset of left mild hemiparesis. Over the past 10 years, he had been admitted six times because of recurrent ischemic strokes despite taking antiplatelet medications, direct oral anticoagulants, and statin medications. The etiology of recurrent strokes had not been clearly identified so far and the IA was not examined before. On arrival at the hospital, he had the same blood pressure on both arms. There were no remarkable laboratory findings including coagulation activity and platelet counts. The lipid profile revealed a total cholesterol of $191 \mathrm{mg} / \mathrm{dL}$; a low-density lipoprotein cholesterol (LDL-C) of $130 \mathrm{mg} / \mathrm{dL}$; a high-density lipoprotein cholesterol (HDL-C) of 


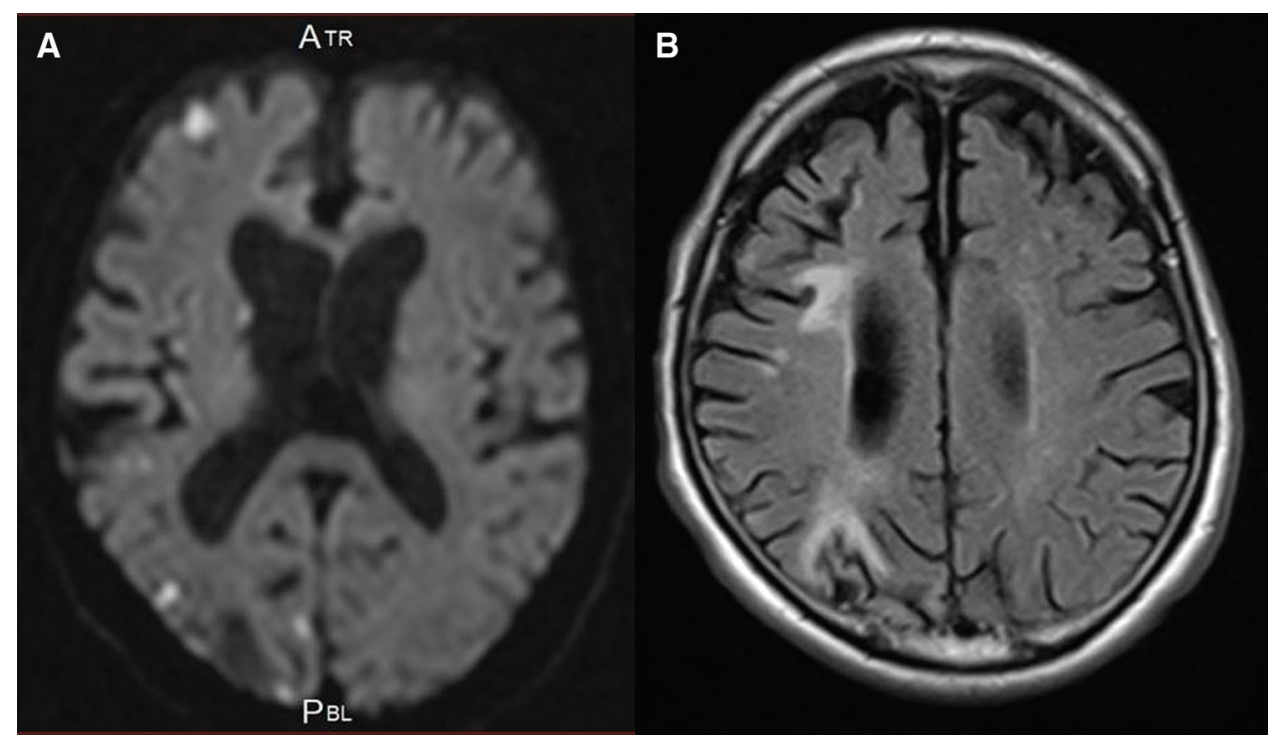

Fig. 1 Preoperative MRI. (A) An axial DWI shows several spotty high-intensity lesions in the right cerebral hemisphere, indicating fresh ischemic lesions. (B) An axial FLAIR image shows several high-intensity lesions in the right cerebral hemisphere, indicating old ischemic lesions. DWI: diffusion-weighted imaging, FLAIR: fluid attenuation inversion recovery, MRI: magnetic resonance imaging.

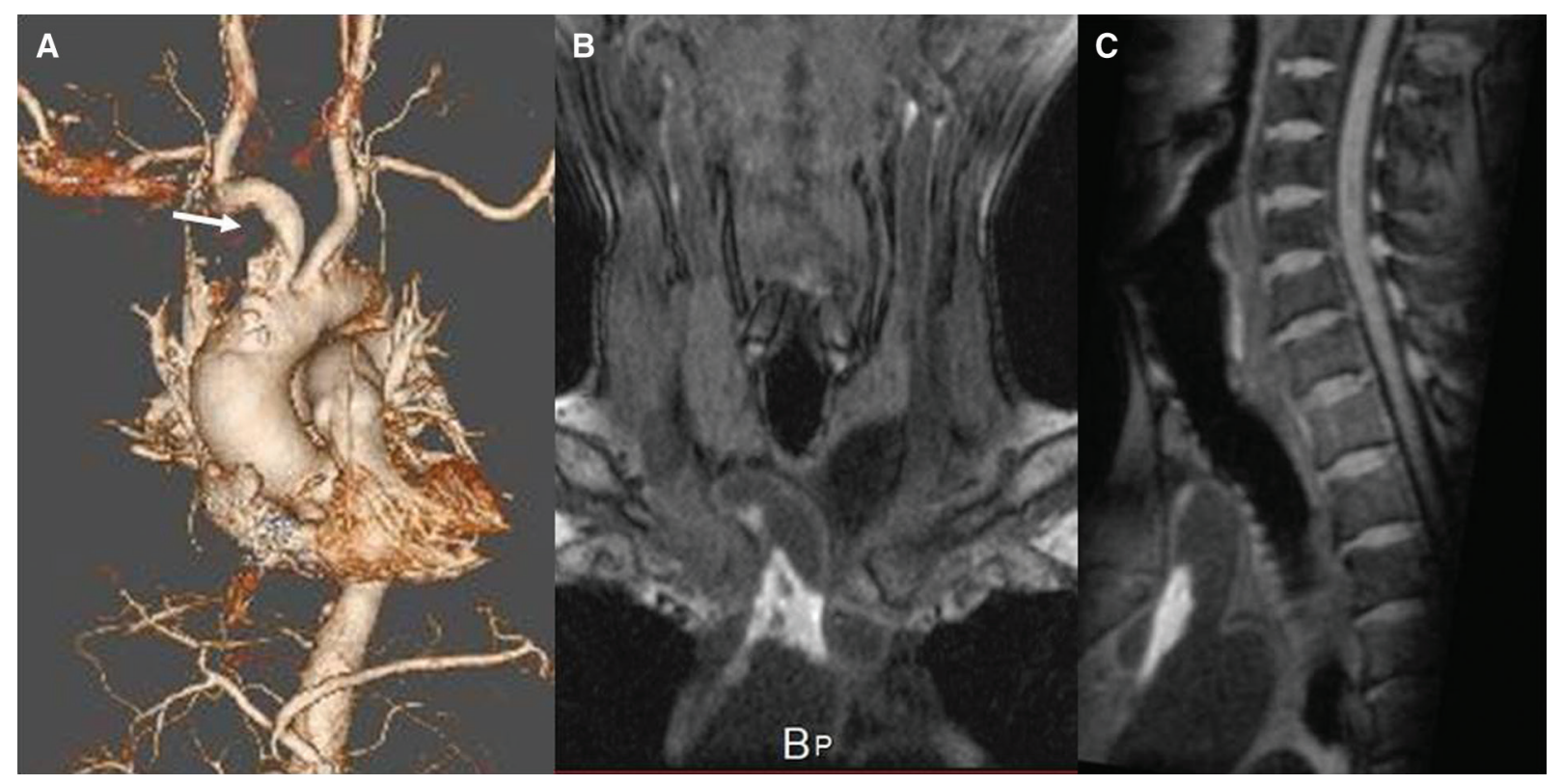

Fig. 2 Preoperative vascular imaging. (A) Three-dimensional reconstruction CT image with contrast enhancement shows a contrast defect in IA, indicating a stenotic lesion. (B) Coronal T1-weighted image with MPRAGE shows a high-intensity plaque in the IA, indicating an intramural hematoma. (C) Sagittal T1-weighted image along the long axis of the IA with MRRAGE shows the same lesion located in the anterior wall of the IA. CT: computed tomography, IA: innominate artery, MPRAGE: magnetization-prepared rapid acquisition gradient echo.

$38 \mathrm{mg} / \mathrm{dL}$; and LDL/HDL ratio of 3.4 and triglycerides of $102 \mathrm{mg} / \mathrm{dL}$. Magnetic resonance imaging (MRI) of the brain showed new ischemic lesions on the diffusion-weighted images as well as old ischemic lesions on the fluid-attenuated inversion recovery images in the right hemisphere (Figs. 1A and 1B). Magnetic resonance angiography of the head and neck revealed no significant stenosis responsible for recurrent ischemic strokes. No abnormalities were detected on electrocardiography, Holter 


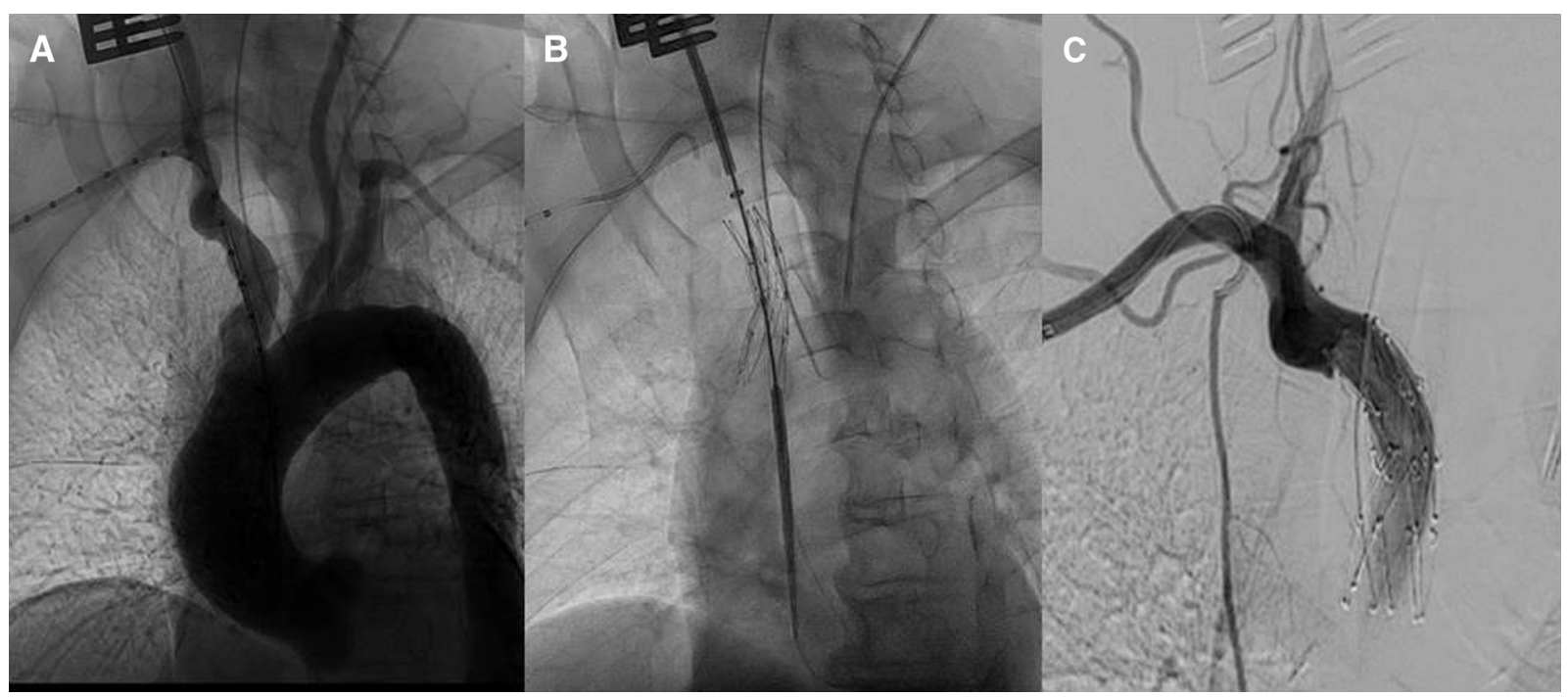

Fig. 3 Intraoperative aortography. (A) The degree of stenosis and the length of the lesion were measured to decide on the appropriate size of the stent graft. (B) The stent graft was deployed in the IA under the fluoroscopic guidance. (C) The IA lesion was properly covered with the stent graft. IA: innominate artery.

electrography, or echocardiography. Computed tomography (CT) of the thorax with contrast enhancement revealed a gross intramural hematoma in the IA (Fig. 2A). MRI using the magnetization-prepared rapid acquisition gradient echo (MPRAGE) sequence revealed a high-intensity lesion on T1 in the corresponding intramural plaque (Figs. $2 \mathrm{~B}$ and $2 \mathrm{C}$ ), indicating a lipid-rich, large and fragile plaque, which was thought to be the cause of the repeated embolic strokes. We decided to use endovascular stent grafting to seal the plaque with distal protection to avoid embolic complications during the procedure, hereby prevent further ischemic strokes. The AFX Endovascular AAA Stent Graft System (Endologix, Inc., Irvine, CA, USA) was employed in terms of plaque sealing and size of the IA and informed consent regarding off label use of this stent graft device for the IA was obtained from the patient and his wife.

\section{Procedure}

Surgery was performed under general anesthesia. First, the right brachial artery as well as the right common carotid artery (CCA) was exposed to avoid postoperative puncture site complications such as hematoma and pseudoaneurysm formation. A 7-French 25-cm double lumen sheath (Medikit, Tokyo, Japan) was inserted into the right brachial artery while a 5-French 10-cm sheath (Medikit) was inserted into the right CCA. For embolic protection, the PercuSurge GuardWire (Medtronic, Minneapolis, MN, USA) was introduced from one lumen of the 7-French double lumen sheath into the right vertebral artery
(VA) and inflated. Meanwhile, the CCA was temporarily clamped with vascular tape just distal to the 5-French sheath whenever a device passed through the IA lesion. Once these distal protections were prepared, a $260 \mathrm{~cm}$ Amplatz Super Stiff Guidewire (Boston Scientific, Boston, MA, USA) was introduced from the CCA sheath into the ascending aorta to deploy the stent graft. A pigtail catheter (Merit Medical, South Jordan, UT, USA) was navigated through the other lumen of the 7-French double lumen sheath into the ascending aorta for the aortography. The degree of stenosis and the length of the lesion were measured with the aortography (Fig. 3A), and the decision was made to use the AFX Endovascular AAA System limb extension stent graft with a diameter of $20 \mathrm{~mm}$ and length of $55 \mathrm{~mm}$. The pigtail catheter inserted from 7-French double lumen sheath was pulled back to the subclavian artery. A stent graft delivery sheath was advanced over the Amplatz Super Stiff Guidewire. From the delivery sheath, the stent graft was deployed in the IA under fluoroscopic guidance (Fig. 3B). Aortography was used to confirm that the IA lesion was covered with the stent graft (Fig. 3C). The distal protections of the CCA and VA were reopened while blood was aspirated until no debris was observed. The wounds were closed and the general anesthesia was discontinued.

\section{Postoperative course}

No neurological complication was observed clinically or radiologically. The patient (with modified Rankin Scale of 3) was transferred to a nursing home, and 


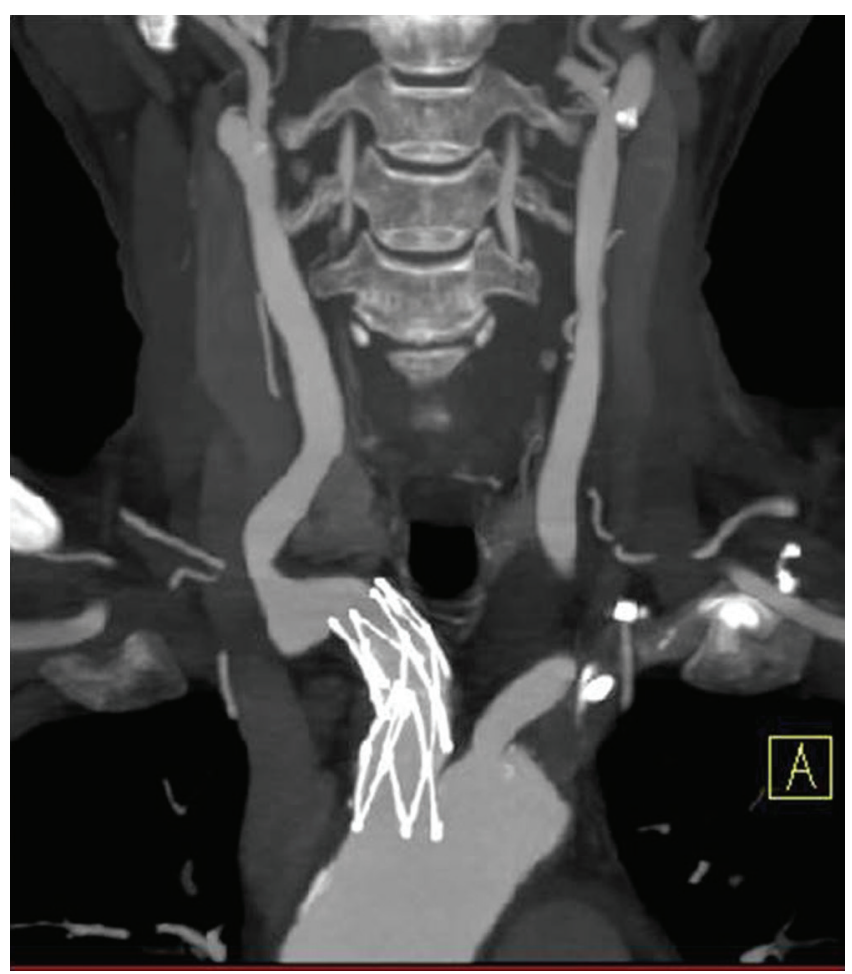

Fig. 4 Postoperative thoracic CT angiography at 8 months shows the properly opened stent graft and no evidence of restenosis or in-stent thrombosis. CT: computed tomography.

followed up at our outpatient clinic with neurological examination every 3 months and MRI every 6 months. After 2 years of follow-up, no recurrent strokes have been observed clinically or on MRI. Postoperative CT angiography at 8 months showed no evidence of restenosis or in-stent thrombosis (Fig. 4).

\section{Discussion}

Although embolism from the atheromatous plaque in the IA is recognized as a possible cause of recurrent stroke, only a few cases have been reported. ${ }^{3-6)}$ IA stenosis comprises $0.5-2 \%$ of all extracranial causes of cerebrovascular insufficiency. ${ }^{7}$ However, there have been no reports regarding the reasons that explain a low incidence of IA atheromatous lesions causing stroke to date. It may be related to anatomical differences that the IA supplies blood to not only the brain but also the right upper extremity, compared to the carotid artery or other intracranial arteries. However, more importantly, it is assumed that the IA is not routinely examined for the stroke work-up in a daily clinical practice and the risk of embolic stroke originating from IA atheromatous plaque could be often overlooked or under-detected. ${ }^{3)}$ Thus, it is important to keep in mind that a thorough systematic search for the embolic sources may be necessary and that the aorta and its main branches should be recognized as possible cause of the embolic stroke. ${ }^{3)}$

In carotid atherosclerotic lesions, vascular imaging such as the evaluation for plaque vulnerability with MRI has been well studied. ${ }^{8,9)} \mathrm{A}$ variety of T1-weighted sequences including MPRAGE, time of flight, and conventional sequences can be used to assess plaque instability. Of these, high intensity on MRI T1-weighted images using the MPRAGE sequence is useful for identifying lipid-rich and fragile plaques, which carry a high risk of artery-to-artery embolism. We applied these plaque imaging techniques to the thoracic aorta and its main branches. MRI using the MPRAGE sequences and CT with contrast enhancement in the thorax clearly demonstrated an intramural large fragile plaque in the IA, which we determined to be the lesion responsible for the recurrent ischemic strokes.

Managing risk factor controls including hypertension, dyslipidemia, diabetes, cigarette smoking, and so on play an important role in preventing stroke recurrence of atherosclerotic origin, in addition to antithrombotic medications. ${ }^{10,11)} \mathrm{He}$ has a long history of hyperlipidemia and his LDL-C level on admission was $130 \mathrm{mg} / \mathrm{dL}$ despite of taking statin medications. The Treat Stroke to Target trial revealed that after an ischemic stroke or TIA with evidence of atherosclerosis, patients who had a target LDL cholesterol level of less than $70 \mathrm{mg}$ per deciliter had a lower risk of subsequent cardiovascular events than those who had a target range of $90 \mathrm{mg}$ to $110 \mathrm{mg}$ per deciliter. ${ }^{12)}$ Moreover, the subanalysis of the JELIS trial revealed administration of highly purified eicosapentaenoic acid (EPA) appeared to reduce the risk of recurrent stroke in a Japanese population of hypercholesterolemic patients receiving low-dose statin therapy. ${ }^{13)}$ Therefore, if IA atheromatous plaque lesion had been detected earlier, stricter control of LDL-C as well as highly purified EPA administration should have been considered.

There have been some case series addressing stent placement for the IA stenotic lesions. ${ }^{7,14,15)}$ However, symptoms in the majority of previous case series were hemodynamic compromise such as upper limb claudication or upper limb arterial pressure asymmetry while some are even without any clinical symptoms. The treatment indications were varied. More importantly, plaque characteristics (calcified, fragile, or fibrous), identified through vascular imaging, were not evaluated. Various types of stents (balloon expandable bare stents, self-expandable bare stents, and balloon expandable covered stents) were used, although the rationale behind selecting those various 
types of stents were not detailed. In the present case, with recurrent strokes due to a gross fragile plaque, we selected the AFX stent graft device as being the most suitable from the standpoints of its plaquesealing capacity and the vessel diameter of the IA (larger and shorter than other supraaortic branch arteries). ${ }^{7,15)}$ The self-expandable AFX stent graft device is known for its endoskeleton design and active sealing structure in which the propriety expanded polytetrafluoroethylene (ePTFE) graft material is externally mounted. This stent provides weak radial force and, thereby, avoids putting excessive stress on a fragile plaque. ${ }^{16)}$ This stent also provides conformability for the irregularities of the vessel wall. In addition, open surgical CCA clamping as well as balloon protection for right VA achieved no ischemic complication. Two years after the stent grafting, the patient has not experienced any recurrence of stroke. The success of the treatment in the case presented here provides valuable insights for establishing the optimal treatment indication, strategy, and preferred type of stents for IA atheromatous plaque lesion.

\section{Acknowledgments}

The authors thank Dr. Alexander Zaboronok, Department of Neurosurgery, and Thomas Mayers of the Medical English Communications Center of the Faculty of Medicine, University of Tsukuba, for manuscript revision.

\section{Conflicts of Interest Disclosure}

The authors declare that there is no conflict of interest.

\section{References}

1) Sardar P, Chatterjee S, Aronow HD, et al.: Carotid artery stenting versus endarterectomy for stroke prevention: a meta-analysis of clinical trials. $J \mathrm{Am}$ Coll Cardiol 69: 2266-2275, 2017

2) de Borst GJ: Innominate artery stenting: the continuing saga of "who, when, and how"? J Endovasc Ther 26: 391-393, 2019

3) Moustafa R, Antoun NM, Coulden RA, Warburton EA, Baron JC. Stroke attributable to a calcific embolus from the brachiocephalic trunk. Stroke 37: e6-8, 2006

4) Nakajima M, Yasaka M, Minematsu K: Mobile thrombus from a ruptured plaque in the brachiocephalic artery. J Stroke Cerebrovasc Dis 17: 423-425, 2008

5) Oishi Y, Hirahara N, Takaseya T, Kawara T, Yasaka M, Morita S: Graft replacement for massive embolic source in brachiocephalic artery. Asian Cardiovasc Thorac Ann 16: e58-e59, 2008

6) Watari $M$, Nakajima $M$, Nishitomi-Izumida $M$, Inatomi Y, Yonehara T, Hirano T: Dynamic migration of a mobile plaque from the brachiocephalic artery detected by ultrasonography. Echocardiography 30: e28-e29, 2013

7) Paukovits TM, Lukács L, Bérczi V, Hirschberg K, Nemes B, Hüttl K: Percutaneous endovascular treatment of innominate artery lesions: a single-centre experience on 77 lesions. Eur J Vasc Endovasc Surg 40: 35-43, 2010

8) Hatsukami TS, Yuan C: MRI in the early identification and classification of high-risk atherosclerotic carotid plaques. Imaging Med 2: 63-75, 2010

9) Gupta A, Baradaran H, Schweitzer AD, et al.: Carotid plaque MRI and stroke risk: a systematic review and meta-analysis. Stroke 44: 3071-3077, 2013

10) Kernan WN, Ovbiagele B, Black HR, et al.: Guidelines for the prevention of stroke in patients with stroke and transient ischemic attack: a guideline for healthcare professionals from the American Heart Association/American Stroke Association. Stroke 45: 2160-2236, 2014

11) Hackam DG, Hegele RA: Cholesterol lowering and prevention of stroke. Stroke 50: 537-541, 2019

12) Amarenco P, Kim JS, Labreuche J, et al.: A comparison of two LDL cholesterol targets after ischemic stroke. N Engl J Med 382: 9, 2020

13) Tanaka K, Ishikawa Y, Yokoyama M, et al.: Reduction in the recurrence of stroke by eicosapentaenoic acid for hypercholesterolemic patients: subanalysis of the JELIS trial. Stroke 39: 2052-2058, 2008

14) Ammi M, Henni S, Salomon Du Mont L, et al.: Lower rate of restenosis and reinterventions with covered vs bare metal stents following innominate artery stenting. J Endovasc Ther 26: 385-390, 2019

15) Mordasini P, Gralla J, Do DD, et al.: Percutaneous and open retrograde endovascular stenting of symptomatic high-grade innominate artery stenosis: technique and follow-up. AJNR Am J Neuroradiol 32: 1726-1731, 2011

16) Diethrich EB: Novel sealing concept in the Endologix AFX unibody stent-graft. J Cardiovasc Surg (Torino) 55: 93-102, 2014

Corresponding author: Tomosato Yamazaki, MD, PhD Department of Neurosurgery, National Hospital Organization, Mito Medical Center, 280 Sakuranosato, Ibaraki, Ibaraki 311-3193, Japan.

e-mail: tysato@hotmail.co.jp 